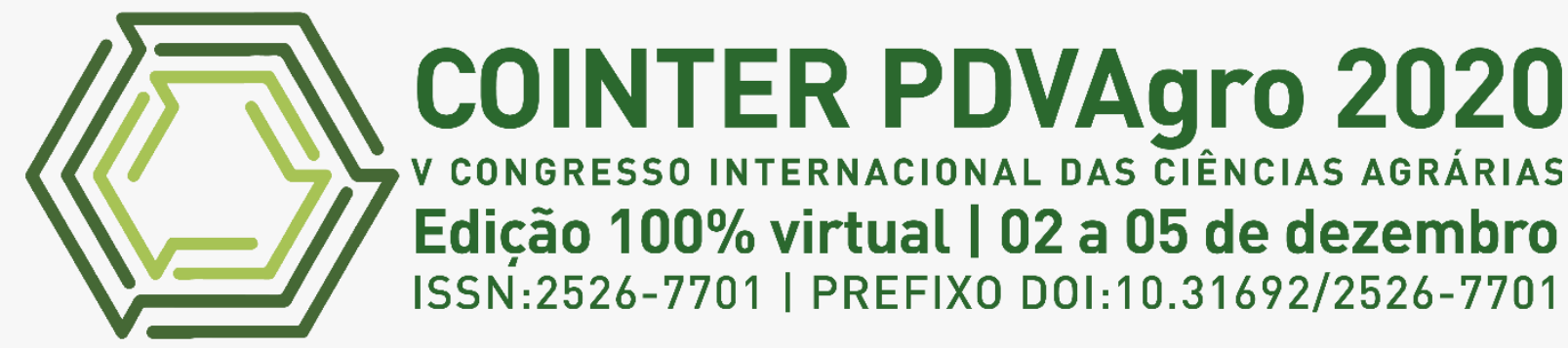

\title{
CONVIVÊNCIA COM O SEMIÁRIDO NO BRASIL, INOVAÇÕES TECNOLÓGICAS COM ARDUINO
}

\section{VIVIENDO CON EL SEMIÁRIDO EN BRASIL, INNOVACIONES TECNOLÓGICAS CON ARDUINO}

\section{LIVING WITH THE SEMI-ARID IN BRAZIL, TECHNOLOGICAL INNOVATIONS WITH ARDUINO}

\author{
Apresentação: Comunicação Oral \\ Luany Emanuella Araujo Marciano ${ }^{1}$; Antônio Marcos Azevedo Batista ${ }^{2}$; Filipe Mendonça de Lima ${ }^{3}$ Leandro \\ Ricardo Rodrigues de Lucena ${ }^{4}$; Moacyr Cunha Filho ${ }^{5}$
}

DOI: https://doi.org/10.31692/2526-7701.VCOINTERPDVAgro.0439

\begin{abstract}
RESUMO
O objetivo deste trabalho é identificar e analisar estudos realizados a nível mundo, Brasil e local, utilizando a aplicação do Arduino em agroecossistemas sustentáveis. Á vista disso, o presente artigo detalha os resultados de uma revisão sistemática conduzida por um protocolo de pesquisa, o qual foi formalizado e executado criteriosamente. Para viabilizar a documentação e registro de todas as etapas da revisão sistemática, foram elaborados quatro formulários com o objetivo de colaborar com a extração e sumarização dos dados. As populações consideradas foram as pesquisas que aplicam o Arduino em agroecossistemas sustentáveis e quanto ao método de busca de fontes, foram acessadas via web, portanto, no âmbito desta revisão, a busca manual não foi considerada. Para o procedimento da pesquisa bibliográfica para seleção de estudos primários, foi utilizada a combinação de síntese de busca "Arduino" AND sustainability; "Uso do Arduino em agroecossistemas sustentáveis" e "use of Arduino in agroecosystems in the semiarid region"; essas sintaxes foram mais adequadas após tentativas com outros termos do tema como "application of Arduino in agroecosystems" e "Arduino" AND agroecosystems. A partir da aplicação das sínteses de busca, foram encontrados o quantitativo de 50 artigos na Scopus, 32 artigos na Web of Science, 39 em português e 149 em inglês no Google Scholar. Os resultados foram expostos e discutidos de acordo com a análise bibliométrica dos dados coletados. Com relação ao uso do Arduino em agroecossistemas, foram encontrados pesquisas que utilizaram desde sistemas de monitoramento de carga elétrica sem fio para zonas rurais, monitoramento do conteúdo de água do solo, sistemas de rastreamento de animais, índice de área foliar até sistemas de irrigação. Com a busca bibliográfica foi possível ter contato com artigos da literatura nacional e internacional na área das Ciências Agrárias, com ênfase no uso de tecnologias aplicadas no setor agropecuário.
\end{abstract}

Palavras-Chave: sistema embarcado, sustentabilidade, territórios rurais.

\section{RESUMEN}

\footnotetext{
${ }^{1}$ Mestrado em Biometria e Estatística Aplicada, UFRPE, marcianoluany@ gmail.com

${ }^{2}$ Graduação em Agronomia, UFPB, aazevedobatista@gmail.com

${ }^{3}$ Doutorado em Biometria e Estatística Aplicada, UFRPE, professorfilipelima@gmail.com

${ }^{4}$ Professor Adjunto, UFRPE/UAST, Unidade Acadêmica de Serra Talhada leandroricardo_est@yahoo.com.br

${ }^{5}$ Professor Associado, UFRPE, moacyr.cunhafo@ufrpe.br
} 
El objetivo de este trabajo es identificar y analizar estudios realizados a nivel mundial, brasileño y local, utilizando la aplicación de Arduino en agroecosistemas sostenibles. En vista de esto, este artículo detalla los resultados de una revisión sistemática realizada por un protocolo de investigación que fue formalizado y cuidadosamente ejecutado. Con el fin de hacer factible la documentación y registro de todas las etapas de la revisión sistemática, se diseñaron cuatro formularios con el objetivo de colaborar con la extracción y resumen de los datos. La población considerada fue la investigación que utiliza Arduino en agroecosistemas sostenibles y en cuanto al método de búsqueda de fuentes, se accede a las mismas vía web, por lo que en el alcance de esta revisión no se consideró la búsqueda manual. Para el procedimiento de búsqueda bibliográfica para la selección de estudios primarios, se utilizó la combinación de síntesis de búsqueda "Arduino" Y sostenibilidad; "Uso de Arduino en agroecosistemas sostenibles" y "Uso de Arduino en agroecosistemas en la región semiárida"; estas sintaxis resultaron más apropiadas luego de intentos con otros términos del tema como "aplicación de Arduino en agroecosistemas" y "Arduino" Y agroecosistemas. De la aplicación de las síntesis de búsqueda, se encontraron 50 artículos en Scopus, 32 artículos en Web of Science, 39 en portugués y 149 en inglés en Google Scholar. Los resultados fueron expuestos y discutidos de acuerdo al análisis bibliométrico de los datos recolectados. Con respecto al uso de Arduino en agroecosistemas, se encontraron investigaciones que utilizaron sistemas inalámbricos de monitoreo de carga eléctrica para áreas rurales, monitoreo del contenido de agua del suelo, sistemas de rastreo de animales, índice de área foliar y sistemas de riego. Con la búsqueda bibliográfica fue posible tener contacto con artículos de literatura nacional e internacional en el área de Ciencias Agrarias, con énfasis en el uso de tecnologías aplicadas en el sector agrícola.

Palabras Clave: sistema integrado, sostenibilidad, territorios rurales.

\section{ABSTRACT}

The objective of this work is to identify and analyze studies carried out at the world, Brazil, and the local level, using the application of Arduino in sustainable agroecosystems. Given this, this article details the results of a systematic review conducted by a research protocol that was formalized and carefully executed. To make the documentation and registration of all stages of the systematic review feasible, four forms were designed to collaborate with the extraction and summarization of data. The population is considered as the research that uses Arduino in sustainable agroecosystems and as for the method of searching for sources, they were accessed via the web, therefore, in the scope of this review, a manual search was not considered. For the bibliographic research procedure for selecting primary studies, the combination of search synthesis "Arduino" AND sustainability was used; "Use of Arduino in sustainable agroecosystems" and "use of Arduino in agroecosystems in the semiarid region"; these syntaxes were more appropriate after attempts with other terms of the theme such as "application of Arduino in agroecosystems" and "Arduino" AND agroecosystems From the application of the search syntheses, 50 articles were found in Scopus, 32 articles in Web of Science, 39 in Portuguese and 149 in English in Google Scholar. The results were exposed and discussed according to the bibliometric analysis of the collected data. Regarding the use of Arduino in agroecosystems, researches were found that used wireless electric charge monitoring systems for rural areas, monitoring of soil water content, animal tracking systems, leaf area index, and irrigation systems. With the bibliographic search, it was possible to have contact with articles of national and international literature in the area of Agrarian Sciences, with emphasis on the use of technologies applied in the agricultural sector.

Keywords: embedded system, rural territories, sustainability.

\section{INTRODUÇÃOO}

O clima semiárido é caracterizado por longos períodos de estiagem, temperaturas superiores a $20^{\circ} \mathrm{C} / a n o$, chuvas escassas e mal distribuídas com precipitações entre 280 e 800 mm/ano. Este clima é predominante na região Nordeste do Brasil, podendo também ser encontrado em continentes como Europa, Ásia e América do Norte. O período de chuva varia muito durante o ano e depende de fatores como, dinâmica da atmosférica, relevo, ventos e fluxo 
de massas de ar, tais características climáticas, pedológicas e hidrológicas dificultam o desenvolvimento da agricultura na região (ARAUJO, 2011).

Os solos característicos da região Nordeste com clima predominantemente semiárido são solos rasos, compactados, com predomínio de areia quartzosa e pela presença de camadas distintas, resultante do material de origem. Além de baixa capacidade de retenção de água e elevada suscetibilidade a inundações (BATISTA et al., 2017). Sabendo disso, torna-se necessário implementar pastagens que se adequem a esse tipo de clima para assim suprir as necessidades dos rebanhos presentes na região.

Com isso, a busca por tecnologias na agricultura tem sido foco de pesquisa nos últimos anos por demanda nos produtos agrícolas, essas desempenham um papel decisivo no atendimento às demandas externas e internas. Um ambiente tecnológico tende a influenciar pequenos e grandes produtores, por apresentar inovação e economia de recursos com seu uso em áreas rurais. Porém, os produtores enfrentam dificuldades para assimilar modelos de negócio, sistemas econômicos e tecnologias avançadas. Mesmo com dificuldades, a inovação tecnológica na agricultura tem sido importante para um aumento na produção agrícola (PETRY et al., 2019).

A inserção de tecnologia na agricultura tem mostrado uma forte correlação com o aumento da produção. A tecnologia induzida pelos investimentos em pesquisa, desenvolvimento, assistência técnica e informações tem sido eficaz e necessária para os produtores rurais. Portanto, com o desenvolvimento e a adoção de tecnologias, o sucesso da agricultura tem crescido no país, atrelado a políticas científicas e de desenvolvimento rural que são levadas por profissionais do setor (EKBOIR, 2003).

Segundo Gaviola e Costa (2011), o uso de tecnologia no campo é essencial para qualquer produtor, seja pequeno ou grande, no entanto, nem todos os agricultores podem ter acesso a esse recurso em sua propriedade, por isso, tecnologia de baixo custo e eficiente como o arduíno, tem proporcionado resultados satisfatórios aos produtores.

$\mathrm{O}$ arduino é usado como uma ferramenta de baixo custo pelos produtores que visam facilidade e automação do trabalho e eficácia no desenvolvimento. Essa tecnologia tem o poder de controlar diversos componentes que emitem dados, mostrado ser muito eficiente em áreas na agricultura com o uso de sensores, motores, dispositivos de GPS, dentre outros (ALMEIDA et al., 2019).

Almeida et al. (2019) utilizaram microcontroladores, que desempenham funções especificas para a medição do $\mathrm{pH}$ do solo, utilizando um aplicativo conectado com um sistema de arduino que passa todas as informações referente ao $\mathrm{pH}$ através de comandos específicos no 


\section{CONVIVÊNCIA COM O SEMIÁRIDO NO BRASIL, INOVAÇÕES TECNOLÓGICAS COM}

aplicativo, e mostra com eficácia, os valores máximos e mínimos de $\mathrm{pH}$ do solo e suas devidas colorações. Além dessa aplicação do arduíno no setor agrário, existem outras diversas, como, irrigadores automáticos, sistemas de controle da água do solo, sistemas de rastreamento de animais, índice de área foliar e programas de iluminação.

Dessa forma, o objetivo deste trabalho é identificar e analisar estudos realizados a nível mundo, Brasil e local, utilizando a aplicação do arduino em agroecossistemas sustentáveis por meio de uma revisão sistemática. Á vista disso, o presente artigo detalha os resultados de uma revisão sistemática conduzida por um protocolo de pesquisa que foi formalizado e executado criteriosamente.

\section{FUNDAMENTAÇÃO TEÓRICA}

O arduíno surgiu inicialmente na Itália no ano de 2005, com o professor Massimo Banzi, o qual tinha o objetivo de criar um dispositivo para controlar projetos através de programação de computadores, de forma simples e barata, além do mais, a sua aplicabilidade é ilimitada (SILVA et al., 2014).

O arduino é uma plataforma única de controle eletrônico, que possui um microcontrolador. É rápido, prático e de baixo custo, sendo assim, acessível por diversas pessoas e empresas no mundo, utilizado para diversos fins, na indústria e na agropecuária como uma ferramenta de tecnologia, para controlar e facilitar os trabalhos desenvolvidos. Ele permite enviar pulsos em um dado tempo, na frequência desejada para permitir controle no período certo. A programação do arduino para realizar as funções desejadas, é necessário a instalação do seu IDE, que é um software livre que permite programar na linguagem desejada (MCROBERTS, 2018).

Existem modelos de placas de arduíno diferentes disponíveis no mercado, que se apresentam de maneira geral em Nano (pequeno porte), Uno (médio porte) e Mega (grande porte), todas possuem um microcontrolador, conexão USB e pinos digitais de entrada e saída, mas apresentam algumas especificidades de aumento de funcionalidades ou maior capacidade de memória, que são fatores importantes na decisão de qual placa de arduíno escolher para desenvolvimento de projetos (PONTES, 2014).

O uso do arduíno é ideal para criação de dispositivos que possibilitam a interação com o ambiente, realizando a coleta de dados como temperatura, luz e som, permitindo assim sua aplicação no campo, quando usados com aparelho eletrônico portátil como laptop, notebook ou smartphone (SOUZA et al., 2011).

Como exemplo do uso de ferramenta agropecuária a partir do arduino, tem-se o irrigador 
automático, que é um sistema sustentável e de baixo custo com microcontroladores, que podem ser programados e instalados em qualquer meio, podendo também auxiliar para produções agroecológicas reduzindo as dificuldades e economizando água, que é um bem tão precioso e valioso para agricultura (CUNHA e ROCHA, 2016).

O Arduino é utilizado em sistemas automáticos na irrigação por válvulas, através de micro comandos pela plataforma de software, reduzindo a quantidade de água e contribuindo para um ambiente mais sustentável (PEREIRA et al, 2020).

$\mathrm{Na}$ agropecuária de automação de sistema, visando reduzir custos e aumentar o bemestar animal, uma tecnologia de baixo custo empregada foi um microcontrolador, usando o sistema arduino para controlar e monitorar um programa de iluminação em uma instalação para aves de corte, em função da idade das aves (BORGES et al., 2019).

Dessa forma, com a ampla possibilidade de utilização do arduíno em agroecossistemas, seu baixo custo, facilidade de implantação e utilização por pessoas especializadas ou não, tornase viável a inserção dessa tecnologia para pequenos e grandes produtores rurais, aumentando assim a produtividade, facilitando a mão de obra e reduzindo custos.

\section{METODOLOGIA}

Esta Revisão Sistemática busca identificar e analisar estudos realizados, utilizando tecnologia do Arduino aplicada em agroecossistemas sustentáveis. Espera-se alcançar, por meio desta revisão sistemática, dados bibliométricos capazes de dar um panorama de como essas metodologias estão sendo aplicadas, em que territórios, quais populações são influenciadas pelo estudo, quais países e revistas ou jornais publicam mais sobre essa temática.

O planejamento e protocolo da revisão sistemática foi executado de acordo com os estágios e fases propostos por Tranfield; Denyer; Smart (2003), e baseado no modelo de protocolo apresentado por Biolchini et al. (2007).

O protocolo de Revisão Sistemática de Literatura, teve como finalidade, buscar respostas e esclarecimentos para algumas questões de pesquisa, tais como: quais são as aplicações do arduino em agroecossistemas sustentáveis no mundo; Como esses estudos estão distribuídos geograficamente pelo mundo; qual o perfil bibliométrico dos estudos encontrados; quais os territórios, populações e atividades impactadas pelos estudos; e quais os desafios e dificuldades encontrados durante os estudos com relação ao método.

A população considerada foram as pesquisas que aplicam o arduino em agroecossistemas sustentáveis. A intervenção considerada foram as aplicações do arduino em sistemas sustentáveis desenvolvidos para agroecossistemas. E o resultado considerado foi uma 


\section{CONVIVÊNCIA COM O SEMIÁRIDO NO BRASIL, INOVAÇÕES TECNOLÓGICAS COM}

visão abrangente do perfil das pesquisas que já foram desenvolvidas até o momento, aplicando a metodologia do arduino em sustentabilidade de agroeocossistemas. Foram utilizados como controle as publicações encontradas no Scopus, na Web of Science e no Google Scholar, no tema aplicação do arduino em agroecossistemas sustentáveis, em um intervalo de tempo de 2011 até 2020.

A busca das fontes foi definida de acordo com os critérios de disponibilidade de consulta de pesquisas através da web; presença de mecanismos de busca por meio de palavras-chave; garantia de resultados únicos quando buscados por um mesmo conjunto de palavras-chave; e artigos científicos publicados em periódicos da área de meio ambiente e agrárias, ou interdisciplinar, que contemplem pesquisas aplicando o arduino em agroecossistemas sustentáveis. Quanto ao método de busca de fontes, foram acessadas via web, portanto, no âmbito desta revisão, a busca manual não foi considerada.

As fontes selecionadas foram as bases de dados eletrônicas, disponíveis no portal periódicos CAPES, incluindo documentos indexados por SCOPUS e Web Of Science (SciELO), e Google Scholar, pois ambas as bases possuem critérios de busca avançados e são consolidados na área de pesquisa. Os idiomas incluído nas buscas foram o inglês e português. A escolha do inglês deve-se à sua universalidade e por ser o idioma internacionalmente aceito para a redação de trabalhos científicos. E o português para abranger trabalhos produzidos pela comunidade científica brasileira e acima de tudo, valorizar a produção científica nacional. É importante destacar que foi incluído como filtro de buscas apenas trabalhos publicados entre 2011 e 2020.

Para a seleção dos estudos foram definidos para cada uma das questões de pesquisa critérios de inclusão e exclusão. A escala envolvida nos critérios é nominal envolvendo duas opções: "Incluído" ou "Excluído".

Foram incluídos artigos científicos de periódicos nacionais e internacionais, teses e dissertações disponíveis integralmente em bases de dados científicas em versões online; trabalhos publicados entre 2011 e 2020, que já possuam aprovação pela comunidade científica; e trabalhos que aplicaram o arduino em agroecossistemas sustentáveis.

Foram excluídos trabalhos que não estavam integralmente disponíveis nas versões online e não se caracterizavam como artigos científicos de periódicos, tese ou dissertações; trabalhos que não seguiram com critério e rigor a sua metodologia; e trabalhos que não se encaixaram no tema pesquisado.

Os estudos selecionados mediante cumprimento dos critérios de seleção dos estudos primários foram examinados pelos pesquisadores consoantes os critérios de qualidade 
estabelecidos. O resultado da avaliação da qualidade de cada estudo determina sua inclusão ou exclusão da lista dos estudos de onde serão extraídos os dados.

Após a seleção foi feita a avaliação da qualidade dos estudos primários do trabalho, o qual deve ter sido publicado em periódico com revisão por pares quando se referir a artigos. Após definidos os trabalhos definitivamente incluídos, durante o processo de avaliação da qualidade dos estudos primários, foi feito a leitura na íntegra e detalhadamente.

Com isso, aplicou-se a estratégia de extração dos dados, o revisor fez um resumo em forma de notas de cada um deles, destacando período da pesquisa, território de aplicação da pesquisa, quais dimensões utilizou, quanto e quais indicadores aplicou, conclusões da pesquisa, desafios e dificuldades encontrados ao longo do trabalho.

Foram preenchidos "formulários de extração de dados" para cada texto, considerado válido para a revisão sistemática. Este formulário de extração contém todos os dados bibliográficos da pesquisa, incluindo data de publicação, abstract, entre outros, bem como a síntese do trabalho, redigida pelo pesquisador a fim de nortear as reflexões pessoais e conclusões do estudo.

Para viabilizar a documentação e registro de todas as etapas da revisão sistemática, foram elaborados os seguintes formulários com o objetivo de colaborar com a extração e sumarização dos dados:

I. Formulário de Protocolo da Revisão Sistemática: Registro contendo todo o planejamento da revisão, descrevendo todas as etapas necessárias para a execução do estudo. Neste documento, organizado no Microsoft Word, estão pormenorizados os objetivos da pesquisa, questões de pesquisa, escopo e especificidades da revisão, critérios de busca das fontes, critérios para seleção dos estudos, processo de seleção dos estudos primários, estratégia de extração dos dados, e por fim, sumarização dos resultados e disseminação do conhecimento.

II. Formulário de Condução da Revisão Sistemática: Documento, organizado em Microsoft Excel, contendo os campos para o registro de informações sobre a data de realização da busca, a base de dados onde a busca foi realizada, as combinações de palavras-chave que proporcionaram a busca, operadores booleanos e filtros utilizados, e o quantitativo dos artigos encontrados para cada busca.

III. Formulário de Seleção de Estudos: Formulário, organizado em Microsoft Excel, contendo campos descriminados informando: nome do(s) Autor(es), ano da publicação, título do artigo, nome do Jornal, resumo, objetivo do artigo, objetivo do autor ao escrever o artigo, quais os conceitos abordados, o que o autor traz de novidade para o tema abordado, método, resultados, conclusões do artigo, contribuição para o meu trabalho, observações (Insights para 


\section{CONVIVÊNCIA COM O SEMIÁRIDO NO BRASIL, INOVAÇÕES TECNOLÓGICAS COM}

o meu trabalho), critério(s) de inclusão atendido(s), critério(s) de exclusão atendido(s), status do artigo para a fase de extração de dados (pendente, incluído ou excluído).

IV. Formulário de Extração de Dados: Formulário individual para cada obra incluída no estudo, organizado em Microsoft Excel, contendo campos para um aprofundamento no tocante ao resumo do artigo, objetivo do estudo, descrição do estudo experimental, resultados do estudo, além de notas adicionais do pesquisador que extraiu os dados. Nesta fase de extração dos dados, foi utilizado um software gratuito, o gerenciador de referências Mendeley, onde foram feitas as extrações e anotações detalhadas de cada trabalho selecionado para o estudo.

A revisão sistemática foi conduzida entre setembro e outubro de 2020, durante um período de 15 dias. Foram selecionados artigos que tiveram os seus dados extraídos e analisados, de acordo com as recomendações previstas no protocolo de revisão.

Para o procedimento da pesquisa bibliográfica para seleção de estudos primários, foi utilizada as combinações de síntese de busca, "Arduino" AND sustainability; "Uso do arduino em agroecossistemas sustentáveis", "Uso do arduino em agroecossistemas no semiárido" e "use of arduino in agroecosystems in the semiarid region"; essas sintaxes foram mais adequadas após tentativas com outros termos do tema como "application of arduino in agroecosystems" "aplicação do arduíno em agroecossistemas sustentáveis" e "Arduino” AND agroecosystems. A partir da aplicação das sínteses de busca, foram encontrados o quantitativo de 50 artigos na Scopus, 32 artigos na Web of Science, 39 em português e 149 em inglês no Google Scholar.

A avaliação da qualidade dos estudos primários (filtragem) foi realizada a partir dos 270 artigos selecionados na fase da pesquisa bibliográfica. Os artigos foram filtrados e organizados em planilha Excel de acordo com os critérios de repetição do artigo em mais de uma base de dados, e o ano de publicação, para a determinação da cronologia de evolução do método. Dessa forma, a partir da amostra de 270 artigos foram selecionados 28 artigos no tema aplicação do arduino em agroecossistemas sustentáveis, em um intervalo de tempo de 2011 até 2020.

Após realização da filtragem, foi aplicada a estratégia de extração dos dados, no qual foi feito um resumo em forma de notas dos artigos, destacando período da pesquisa, território de aplicação da pesquisa, dimensões utilizadas, quanto e quais indicadores aplicou, conclusões da pesquisa, desafios e dificuldades encontrados ao longo do trabalho. Assim a amostra de 28 artigos foi reduzida para uma amostra de nove artigos. 
Figura 1. Fluxograma das etapas da Revisão Sistemática da Literatura

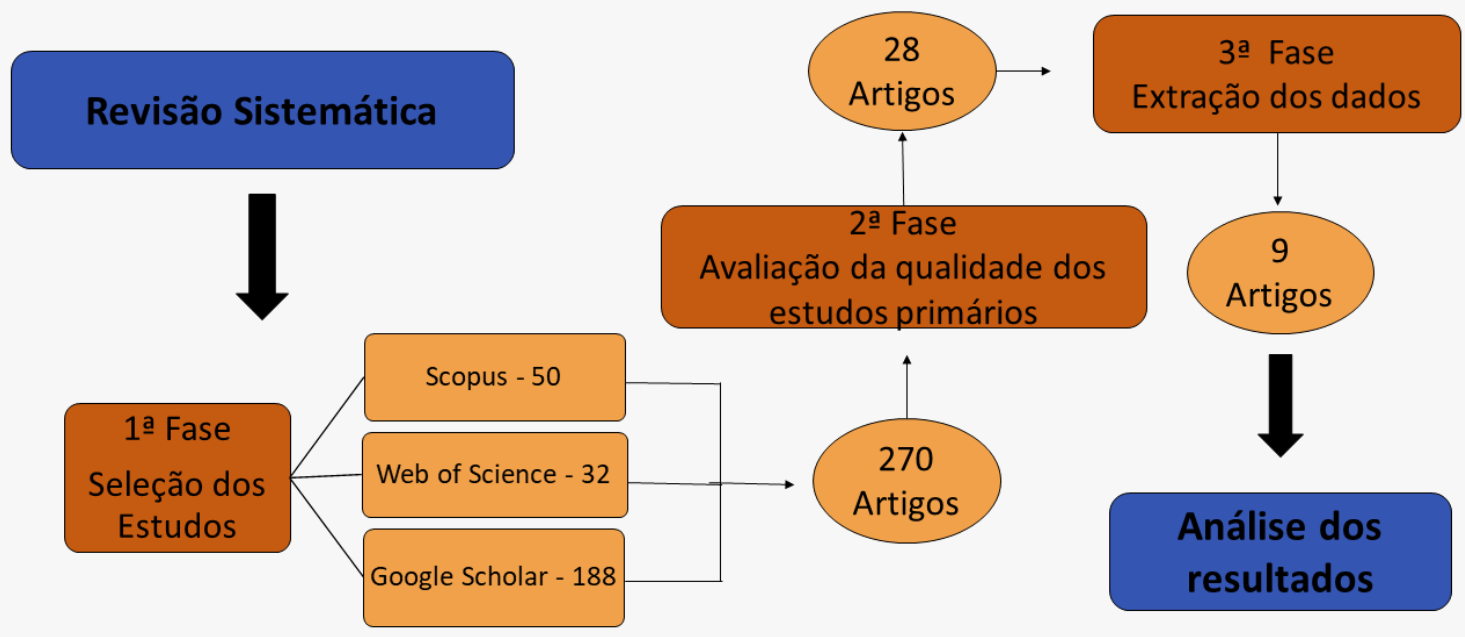

Fonte: Própria (2020)

Algumas fontes reconhecidamente importantes no contexto da pesquisa não foram contempladas pelo protocolo de revisão, tendo em vista que não atendiam aos critérios de seleção de fontes.

A revisão sistemática conduzida, desde sua concepção até a extração dos resultados encontra-se documentada por meio dos seus formulários eletrônicos que se encontram arquivados em pastas da área de trabalhos da pesquisadora principal, backup em HD externo pessoal, e em nuvem de armazenamento forma a permitir sua repetição e auditagem por pesquisadores interessados no estudo.

\section{RESULTADOS E DISCUSSÃO}

Mediante consultas às bases de dados, acerca do uso do arduino em agroecossistemas sustentáveis, destacou-se que os unitermos utilizados, embora indicassem em alguns momentos, artigos repetidos ou revelassem estudos com temas divergentes ao pesquisado. Foi possível selecionar artigos que abrangessem o tema, ou parte dele, para assim haver uma análise dos trabalhos selecionados (Tabela 1). 
CONVIVÊNCIA COM O SEMIÁRIDO NO BRASIL, INOVAÇÕES TECNOLÓGICAS COM

Tabela 1. Estudos do Uso do Arduino em Sistemas Sustentáveis no Meio Rural

\begin{tabular}{cccc}
\hline Autor & Ano & Base de Dados & Periódico \\
\hline McGranahan et al. & 2018 & Google Scholar & Ecology and Evolution \\
Kim et al. & 2019 & Google Scholar & ELSEVIER \\
Di Prima et al. & 2020 & Google Scholar & Journal Water \\
Petros Spachos & 2020 & Google Scholar & Journal IOT \\
Ramadan et al. & 2018 & Scopus & ELSEVIER \\
X. Wang et al. & 2020 & Scopus & Springer \\
X. Wang et al. & 2020 & Scopus & ELSEVIER \\
Bitella et al. & 2014 & Web of Science & Sensors \\
D. Masseroni et al. & 2016 & Web of Science & IRRIGATION AND \\
\end{tabular}

Fonte: Própria (2020)

A base de dados Google Scholar foi a que apresentou maior número de resultados a partir da busca, com 149 artigos em inglês e 39 em português, no entanto a plataforma contempla uma diversidade muito ampla de documentos, como livros, resumos de congressos, boletins, artigos publicados em revistas ou eventos e trabalhos de conclusão de curso, teses e dissertações. Como a intenção do presente trabalho é selecionar artigos publicados em periódicos, teses e dissertações, muitos trabalhos não se encaixaram nos critérios de inclusão. Outra característica observada nessa plataforma foi que ao avançar as páginas de busca, as palavras-chaves não eram todas contempladas e assim os trabalhos diferiam muito da pesquisa.

$\mathrm{Na}$ base de dados Scopus, foram identificados uma quantidade menor de artigos, no geral, esses artigos foram publicados em periódicos de revistas ou jornais. Os artigos abordavam o tema do uso do arduino em agroecossistemas (Figura 2), agricultura sustentável ou uso de tecnologias no meio rural, mas também haviam artigos que abordavam uso de tecnologias com o arduino empregadas no meio urbano.

Figura 2. Arduino em sistema embarcado, utilizado como sensor de umidade em agroecossistemas.

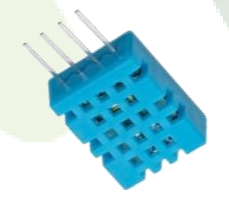

Fonte: www https://br.search.yahoo.com/search

Quanto à base de dados Web of Science, percebeu-se uma grande semelhança com o Scopus, que também abordavam artigos mais voltados ao meio urbano, além disso, havia um número considerável de artigos repetidos.

Com relação ao uso do arduino em agroecossistemas, foram encontrados pesquisas que utilizaram desde sistemas de monitoramento de carga elétrica sem fio em zonas rurais, 
monitoramento do conteúdo de água do solo, sistemas de rastreamento de animais, índice de área foliar até sistemas de irrigação.

Foi também realizado uma síntese com uma abordagem abrangente de acordo com o tema da sistematização dos artigos, para assim conhecer quais as pesquisas estão sendo desenvolvidas com o uso no arduino, que apresenta uma grande multiplicidade de utilização.

No artigo "A Novel Low-Cost Open-Hardware Platform for Monitoring Soil Water Content and Multiple Soil-Air-Vegetation Parameters", os autores BITELLA et al. no ano de 2014, buscaram projetar uma plataforma de hadware livre de baixo custo para medições de multissensores, com água em diferentes profundidades, incluindo temperaturas do ar e do solo. O sistema foi baseado em uma placa microcontroladora arduino programada em Ambiente de Desenvolvimento Integrado (IDE) simples. Esse sistema é importante para a sustentabilidade da agricultura, como a irrigação de precisão, fenotipagem de características de raízes para tolerância à seca, tudo isso colabora para uma economia e redução dos gastos dos produtores, em energia, água, manejo entre outros. O trabalho foi publicado no jornal Sensors na cidade de Pontenza- Itália pela Universidade de Brasilicata.

O artigo "Irrig-Oh: An Open-Hardware Device For Soil Water Potential Monitoring And Irrigation Management" retratou um dispositivo de hardware aberto baseado na tecnologia Arduino, utilizado para desenvolver e permitir o monitoramento contínuo do potencial da água no solo na zona radicular de pêssegos, para apoiar o planejamento da irrigação em escala de campo. A estrutura do dispositivo é flexível e pode ser implantada em diversas culturas visando diminuir a quantidade de água usada nas irrigações na agricultura. $\mathrm{O}$ estudo mostrou um aumento significativo da eficiência do uso da água sem causar redução da quantidade e da qualidade da produção agrícola. Portanto, o uso racional da água contribui para uma maior produção e qualidade, como o uso de microcontroladores em software na plataforma de arduino que determinam a quantidade e o tempo, minimizando assim, o custo com energia, a quantidade de água desperdiçada e a obter melhores resultados nas colheitas. Esse trabalho foi publicado na revista de Irrigation and Drainage, na cidade de Montanaso Lombardo- Itália, por MASSERONI et al. no ano de 2016.

X. WANG et al. no ano de 2020 desenvolveu dois trabalhos sobre o tema. O artigo "Low-cost far-field wireless electrical load monitoring system applied in an off-grid rural area of Tanzania" publicado no Journal Elsevier, e conduzido em uma área rural da Tanzânia, África, teve como sistema proposto uso de placas Arduino para um novo sistema de monitoramento de carga elétrica sem fio de campo distante. Tomando vantagem da tecnologia de radiofrequência (RF), o sistema realiza um ponto a multiponto, em um multicanal de comunicação, alargando 


\section{CONVIVÊNCIA COM O SEMIÁRIDO NO BRASIL, INOVAÇÕES TECNOLÓGICAS COM}

assim, a distância de comunicação. Para permitir o rastreamento da produção de energia e uso de energia fora da rede. Esse método sustentável foi introduzido para desenvolvimento das áreas subdesenvolvidas, contribuindo para a provisão de um fornecimento ininterrupto de energia, buscando melhorias no padrão de vida dos residentes local.

E o artigo "Arduino-based low-cost electrical load tracking system with a long-range mesh network" também publicado no ano de 2020, mas na Springer Journals Archive, se desenvolveu na região da Tanzânia, África. O sistema proposto no trabalho é baseado na placa Arduino, onde essa controla todo o processo de forma rápida e precisa com microcomandos instalados na rede, o que reduz o custo de produção e fornece um simples processo de manufatura. A aplicação do sistema proposto pode promover a sustentabilidade do local estudado, possibilitando as pessoas da zona rural, fornecimento rápido e preciso de energia para desenvolvimento local. Uma fator muito interessante dessa pesquisa é que, ela mostra que essa tecnologia não é limitada por nenhum fator de infraestrutura local ou ambiente geográfico, nem o tipo de objeto de monitoramento, ou seja, pode ser utilizado em qualquer ambiente desde que se tenham as tecnologias necessárias.

No trabalho "Assessment of a livestock GPS collar based on an open-source datalogger informs best practices for logging intensity", fundamentou-se a elaboração de um equipamento de monitoramento de bois e ovelhas em campo, utilizando uma tecnologia de baixo custo baseado na plataforma do arduino, onde utiliza microcontroladores que determinam proximidade entre os animais se estão agrupados, a distância diária total percorrida pelos animais, também pode ser utilizado na localização exata de viagens individuais de animais, com o fim de evitar perdas ou roubos de animais durante a viagem. Para isso foi desenvolvido um datalogger GPS de código aberto baseado no arduino, esse possui uma bateria fixa que alimenta e oferece todo suporte de energia para as coleiras eletrônicas, a vida da bateria pode chegar aos 50 dias de duração. Então para testar o sistema, coletou-se posições em intervalos de 20 segundos em 1 semana de duração, em bovinos e ovinos em pastagens, para rastrear os movimentos dos animais e seu comportamento. Essa pesquisa traz inovação na agropecuária e pode ser expandida para auxiliar os produtores no controle do gado em sistema extensivo. A pesquisa foi publicada na revista Ecology and evolution e se desenvolveu em Dakota do Norte, Estados Unidos, conduzida por MCGRANAHAN et al. em 2018.

Já no artigo "Continuous observation of vegetation canopy dynamics using an integrated low-cost, near-surface remote sensing system", desenvolvido por KIM et al., no ano de 2019. Pode-se avaliar um sistema que coleta, transfere, processa automaticamente imagens da microcâmera e dados de intensidade espectral do LED e fluxos de dados. O sistema consiste 
em um microcomputador arduino com um espectrômetro multiespectral embutido com díodo emissor de luz (LED) e ligação à Internet. Os dados são transferidos para um servidor remoto usando o protocolo de transferência de arquivo. Sendo este, uma novo mecanismo de analisar o índice de área foliar, que é tão importante para compreensão da dinâmica da vegetação em ecossistemas. Esse trabalho foi publicado pelo Journal Elsevier, na República Coreana.

A pesquisa "Design and implementation of a low cost photovoltaic soil moisture monitoring station for irrigation scheduling with different frequency domain analysis probe structures", propõe um plano para o projeto e implementação de duas estruturas de sondas distintas “A" e "B", instaladas para a medição do conteúdo de água no solo em diferentes níveis. Para fazer isso, uma onda quadrada de frequência fixa é transmitida para medir a capacitância do solo em que os sensores da sonda são conectados a um microcontrolador Arduino que também inclui, sensores de umidade, temperatura do ar e temperatura do solo. Portanto, o modelo de sonda "A" pode desempenhar um papel importante no monitoramento do solo umidade em várias profundidades com alto desempenho, precisão e baixo custo. A estação de monitoramento experimental usada para avaliar o a eficiência de ambos os projetos de sondas foi estabelecida em uma zona semiárida mediterrânea no sudeste da Espanha. A estação usada era autônoma, sustentável e precisa em seus resultados, o que motiva e estimula novas pesquisas para melhorar cada vez mais o sistema e auxiliar no desenvolvimento rural agrícola. Esse trabalho foi publicado pelo Journal Elsevier, na Espanha por RAMADAN et al. em 2018.

No artigo "Towards a Low-Cost Precision Viticulture System Using Internet of Things Devices", o foco foi a implementação de sistema de baixo custo para aquisição e monitoramento de dados em tempo real, de um sistema de viticultura de precisão usando um dispositivos IoT em tempo real. Os diferentes componentes do sistema são programados corretamente e a interconexão entre eles é projetada para minimizar o consumo de energia. Nós de sensores sem fio medem a umidade e a temperatura do solo no campo e transmitem informações para a estação base. Então, se as condições forem ideais para a ocorrência de uma doença ou praga, um drone voa imediatamente em direção à área para verificar toda situação com câmeras e microcomandos instalados, conectados a um sistema operacional ligado a base de controle. Esse sistema foi capaz de relatar os dados em tempo real e também localizar o nó que coletou os dados que excedem um limite predefinido com precisão aceitável, então essa pesquisa é bastante relevante no que diz respeito à agricultura de precisão, buscando sustentabilidade agrícola e desenvolvimento rural. Publicado no journal IoT, no Canadá, por SPACHOS em 2020.

O trabalho intitulado, "Soil Hydrology for a Sustainable Land Management: Theory and 


\section{CONVIVÊNCIA COM O SEMIÁRIDO NO BRASIL, INOVAÇÕES TECNOLÓGICAS COM}

Practice”, apresenta algumas contribuições para hidrologia do solo e gestão sustentável da terra. A contribuição desse procedimento foi focada em um método simples e rápido para estimar as propriedades hidráulicas do solo e investigações sobre o tema de erosão da chuva ou o impacto da cobertura vegetal na interação do conteúdo de água do solo. Dentre os métodos descritos na pesquisa, o arduino se destacou por ser um dispositivo de baixo custo. Esse estudo foi relevante pois aumentou o conhecimento sobre a gestão sustentável de ecossistemas específicos, trazendo a importância de se ter conhecimento sobre como a água reage e se comporta no solo. Publicado no Journal Water por Di PRIMA et al. (2020).

Analisando as sínteses descritas, realizadas em diferentes partes do mundo, percebe-se que há uma ampla diversidade na utilização do sistema embarcado, sistema esse, capaz de otimizar diversas áreas da produção rural, reduzir custos e facilitar o trabalho diário. Outro fator observado foi o período de tempo que esses artigos foram desenvolvidos, caracterizando assim um curto período, o que indica que esta é uma tecnologia empregada recentemente no setor agrícola. Mas, a ferramenta apresenta grande potencial de utilização, por ser de baixo custo e facilmente empregada.

Sendo assim, a tecnologia do uso o arduino será de suma importância para o clima Semiárido do Nordeste, tanto para uso na irrigação controlada, medição de água no solo, estudos de análise de área foliar, dentre outros. No entanto, há necessidade de mais estudos com uso do arduino em áreas rurais, especialmente no Brasil, no qual apresenta poucos trabalhos desenvolvidos até o momento.

\section{CONCLUSÕES}

Com a busca bibliográfica foi possível ter contato com artigos da literatura nacional e internacional na área das Ciências Agrárias, com ênfase no uso do arduino aplicadas no setor agropecuário. Permitiu-se, com a definição de um protocolo de revisão sistemática, o estabelecimento de um escopo do estudo, bem como a elaboração do planejamento do cronograma para a condução da revisão, e o monitoramento da pesquisa norteados pelo objetivo da revisão e pelas questões de pesquisa.

Do exposto, destaca-se a importância da abordagem metodológica da revisão da literatura para pesquisas envolvendo o uso do arduino em agroecossistemas, mas, ainda há uma defasagem de pesquisas sobre o tema no mundo, e principalmente no Brasil, necessitando assim de um aprofundamento sobre o assunto, o que possibilitaria um avanço considerável na agropecuária, que é de grande importância para economia brasileira. 


\section{REFERÊNCIAS}

ALMEIDA, B. M.; SOUZA, J. S.; SANCHES, J. R.; OLIVEIRA, J. H. V.; GAMBARATO, V. T. S. Integração de sistema mobile com sistemas que utilizam a plataforma arduino aplicados à agricultura. Tekhne e Logos, v. 10, n. 2, p. 62-75, 2019.

ARAUJO, S. M. S. A Região Semiárida Do Nordeste Do Brasil: Questões Ambientais E Possibilidades De Uso Sustentável Dos Recursos. Rios Eletrônica, v. 5, n. 2, p. 98, 2011.

BATISTA, P. H. D.; ALMEIDA, G. L. P.; TAVARES, U. E.; NASCIMENTO, V. N.; SARMENTO, R. M. Variabilidade espacial de atributos físicos do solo sob colheita mecanizada de capim-buffel no Agreste Pernambucano. Engenharia na Agricultura-Reveng, v. 25, n. 4, p. 307-317, 2017.

BIOLCHINI, J. C. DE A.; MIAN, P. G.; NATALI, A. C. C.; CONTE, T. U.; TRAVASSOS G. H. Scientific research ontology to support systematic review in software engineering. Advanced Engineering Informatics, v. 21, n. 2, p. 133-151, abr. 2007.

BITELLA, G.; ROSSI, R.; BOCHICCHIO, R.; PERNIOLA, M.; AMATO, M. A novel lowcost open-hardware platform for monitoring soil water content and multiple soil-air-vegetation parameters. Sensors, v. 14, n. 10, p. 19639-19659, 2014.

BORGES, P. H. M.; MENDOZA, Z. M. S. H.; MORAIS, P. H. M.; SANTOS, R. L.; CAVALCANTE, C. E.; RUVER, F. S. Automação de baixo custo da iluminação artificial para aves de corte. Revista Eletrônica Competências Digitais para Agricultura Familiar, v. 5, n. 2, p. 20-37, 2019.

CUNHA, K. C. B.; ROCHA, R. V. Automação no processo de irrigação na agricultura familiar com plataforma Arduino. Revista Eletrônica Competências Digitais para Agricultura Familiar, v. 1, n. 2, p. 62-74, 2016.

DI PRIMA, S.; CASTELLINI, M.; RODRIGO-COMINO, J.; CERDÀ, A. Soil Hydrology for a Sustainable Land Management: Theory and Practice. Journal Water, 2020.

EKBOIR, J. M. Research and technology policies in innovation systems: zero tillage in Brazil. Research policy, 32(4), 573-586. 2003.

GAVIOLA, F. R.; COSTA, M. B. P. As múltiplas funções da agricultura familiar: um estudo no assentamento Monte Alegre, região de Araraquara (SP). Revista de Economia e Sociologia Rural, v. 49, n. 2, p. 449-472, 2011.

KIM, J.; RYU, Y.; JIANG, C.; HWANG, Y. Continuous observation of vegetation canopy dynamics using an integrated low-cost, near-surface remote sensing system. Agricultural and Forest Meteorology, Journal Elsevier, v. 264, p. 164-177, 2019.

MASSERONI, D.; FACCHI, A.; DEPOLI, E. V.; RENGA, F. M.; GANDOLFI. Irrigation: An Open Hardware Device for Soil Water Potential Monitoring and Irrigation Management. Irrigation and Drainage, v. 65, n. 5, p. 750-761, 2016.

MCGRANAHAN, D. A.; GEAUMONT, B.; SPIESS, J. W. Assessment of a livestock GPS collar based on an open resource datalogger informs best practices for logging intensity. 
Ecology and evolution, v. 8, n. 11, p. 5649-5660, 2018.

MCROBERTS, M. Arduino básico. 2. ed. São Paulo: Novatec Editora, 2018. 512p.

PEREIRA, F. A. L.; SILVA, S. L.; MEDEIROS, J. F.; FIGUEIRÊDO, V. B.; JÚNIOR, I. S. Q.; NETO, M. P. S. Automação de precisão utilizado arduino e inversos de frequência aplicado a sistemas de irrigação por válvulas. Irriga, v.25, n.1, p. 27-37, 2020.

PETRY, J. F.; SEBASTIÃO, S. A.; MARTINS, E. G.; BARROS, P. B. A. Inovação e difusão de tecnologia na agricultura de várzea na Amazônia. Revista de Administração Contemporânea, v. 23, n. 5, p. 619-635, 2019.

PONTES, A. S. de. Desenvolvimento de um fotômetro LED-Vis portátil e microcontrolado por Arduino. 2014. 95 f. Dissertação (Mestrado em Química) - Universidade Federal da Paraíba, João Pessoa, 2014.

RAMADAN, K. M.; OATES, M. J.; MOLINA-MARTINEZ, J. M.; RUIZ-CANALES, A. Design and implementation of a low cost photovoltaic soil moisture monitoring station for irrigation scheduling with different frequency domain analysis probe structures. Computers and Electronics in Agriculture, Journal Elsevier, v. 148, p. 148-159, 2018.

SILVA, J. L. S.; CAVALCANTE, M. M.; CAMILO, R. S.; GALINDO, A. L.; VIANA, E. C. Plataforma Arduino integrado ao PLX-DAQ: Análise e aprimoramento de sensores com ênfase no LM35. XIV Escola Regional de Computação Bahia, Alagoas e Sergipe (ERBASE). Feira de Santana, BA, 2014.

SOUZA, A. R.; PAIXÃO, A. C.; UZÊDA, D. D.; DIAS, M. A.; DUARTE, S.; AMORIM, H. S. A placa Arduíno: uma opção de baixo custo para experiências de física assistidas pelo PC. Artigo da Revista Brasileira de Ensino de Física, v. 33, n. 1, 1702, p.1-5, 2011.

SPACHOS, P. Towards a Low-Cost Precision Viticulture System Using Internet of Things Devices. Journal IoT, v. 1, n. 1, p. 2, 2020.

TRANFIELD, D.; DENYER, D.; SMART, P. Towards a Methodology for Developing Evidence-Informed Management Knowledge by Means of Systematic Review. British Journal of Management, v. 14, n. 3, p. 207-222, 2003.

WANG, X.; HA, B.; MANONGI, F. A.; JUNG, W.; JANDE, Y. A. C.; AHN, S. Arduino-based low-cost electrical load tracking system with a long-range mesh network. Advances in Manufacturing, Springer p. 1-17, 2020.

WANG, X.; HA, B.; LEE, G.; KIM, H.; YU, J.; RHEE, H.; NJAU, K. N.; JANDE, Y. A C.; AHN, S. Low-cost far-field wireless electrical load monitoring system applied in an off-grid rural area of Tanzania. Sustainable Cities and Society, Journal Elsevier, p. 102209, 2020. 